\title{
The Role of hsa-miR-548I Dysregulation as a Putative Modifier Factor for Glaucoma-Associated FOXC1 Mutations
}

\author{
Cristina Medina-Trillo, ${ }^{1,2}$, José-Daniel Aroca-Aguilar ${ }^{1,2}$, Jesús-José Ferre-Fernández ${ }^{1}$, Carmen-Dora \\ Méndez-Hernández ${ }^{3}$, Laura Morales ${ }^{3}$, Julián García-Feijoo ${ }^{3}$ and Julio Escribano ${ }^{1,2}$
}

\begin{abstract}
${ }^{1}$ Área de Genética, Facultad de Medicina/Instituto de Investigación en Discapacidades Neurológicas (IDINE), Universidad de Castilla-La Mancha, Albacete, Spain; ${ }^{2}$ Cooperative Research Network on Age-Related Ocular Pathology, Visual and Life Quality, Instituto de Salud Carlos III, Madrid, Spain; ${ }^{3}$ Servicio de Oftalmología, Hospital San Carlos, Madrid, SPAIN/Instituto de Investigación Sanitaria del Hospital Clínico San Carlos, Madrid, Spain.
\end{abstract}

\begin{abstract}
Mutations of the FOXC1 transcription factor are involved in a variety of autosomal dominant ocular anterior segment defects, ranging from Axenfeld-Rieger malformations to isolated glaucoma in some patients. In this study we have evaluated the possible role of the c. $* 734 \mathrm{~A}>\mathrm{T} F O X C 1$ variant as a modifier factor of the activity of two FOXC1 mutations previously identified in families primarily affected by dominant glaucoma (haplotypes p.G447_G448insDG-c.*734A>T and p.I126S-c.*734A>T). Previous bioinformatic analyses indicated that the $c * 734 \mathrm{~A}>\mathrm{T}$ variant is located in a potential target sequence for hsa-miR-5481. Co-expression of this miRNA with a reporter cDNA construct in which the wild-type 3'UTR sequence of FOXC1 was fused to the 3'-end of the firefly luciferase coding region, led to approximately $20 \%$ decreased luciferase activity compared to the controls, confirming the presence of a target sequence for hsa-miR-5481. In contrast, this miRNA did not show any effect on the luciferase activity associated with the mutant 3'UTR FOXC1 sequence, showing that it resulted in a loss-of-function of the has-miR-5481 target sequence. In addition, functional evaluation of the two glaucoma-associated haplotypes revealed increased protein levels and transactivation, compared to the corresponding individual coding mutations (approximately 1.2-fold on average). These data support the role of hsa-miR-5481 as a regulator of FOXC1 translation and provide evidence for the c. $* 734 \mathrm{~A}>\mathrm{T}$ variant as a modifier factor for the activity of coding glaucoma-associated FOXC1 mutations.
\end{abstract}

Keywords: congenital glaucoma, dominant glaucoma, FOXC1, glaucoma, miRNA-5481, modifier factor.

\section{INTRODUCTION}

Glaucoma is a highly heterogeneous, irreversible and progressive optic neuropathy produced by the death of the retinal ganglion cells, which results in the degeneration of the optic nerve and eventually in blindness. Raised intraocular pressure (IOP) is the primary risk factor for developing glaucoma. It has been proposed that oxidative stress plays a primary role among the multiple factors involved in glaucoma pathogenesis, affecting both the cells of the trabecular meshwork and the ganglion cells of the retina [1,2]. Most glaucoma cases behave as a late-onset complex disease and in a minority of patients the disease shows an early-onset and follows Mendelian inheritance. The existence of incomplete penetrance and variable expressivity in some Mendelian glaucoma cases indicate the influence of unidentified modifier factors (genetic, stochastic and/or environmental) on the final phenotypic outcome $[3,4]$.

The FOXC1 protein is a member of the FOX class of transcription factors, which regulate, craniofacial, ocular and cardiovascular development [5]. Mutations in the Axenfeld-Rieger $\mathrm{FOXCl}$ gene are involved in different types

\begin{abstract}
*Address correspondence to this author at the Department of Genetics, Faculty of Medicine, Castilla-La Mancha University, Albacete, Spain; Tel: +34 967599200; E-mails: julio.escribano@uclm.es

of autosomal dominant anterior eye segment defects, including syndrome type 3 (ARS; MIM\#602482). Increased risk for glaucoma and variable iris or extra-ocular abnormalities are commonly associated with mutations in this gene [6]. Although most FOXC1 mutations reduce the FOXC1 transactivation ability [7], we have recently described hypermorphic variations associated with dominant glaucoma (Escribano, personal communication). The regulation of $\mathrm{FOXCl}$ gene expression is poorly understood, particularly at the level of translation. Recently, several functional cis-regulatory elements located in the UTRs of this gene have been identified, including a terminal oligopyrimidine tract (Escribano, personal communication), which regulates translation initiation, and target sequences for some miRNAs [8-10]. Disruption of these regulatory sequences alter $\mathrm{FOXC1}$ translation and, therefore, they have the potential to be pathogenic.

It is well documented the role of MicroRNAs (miRNAs) as key regulators of gene expression at the posttranscriptional level in different normal and pathological
\end{abstract}


biological processes $[11,12]$. However, the study of this type of gene regulators in ocular physiology and pathology is very limited. In that sense, it has been shown, for example, that miR-200c regulates trabecular meshwork contraction and intraocular pressure [13] and that miR-204 target genes are involved in apoptosis, reticulum stress and inflammation in human trabecular meshwork cells [14]. In addition, we have reported that the $* 16 \mathrm{~T}>\mathrm{C}$ WDR 36 mutation, identified in one POAG patient, may form part of a predicted target sequence for miR-9 and miR-224 [15].

In this study, we show that FOXC1 mRNA translation is regulated by hsa-miR548-1 and that disruption of its target sequence by the c.*734A $>\mathrm{T}$ FOXCl mutation modifies the activity of two coding mutations of the same gene.

\section{MATERIALS AND METHODS}

To evaluate the effect of the c.*734A > T FOXC1 variant as a modifier factor, two haplotypes (p.G447_G448insDG-c.*734A>T and p.I126S-c.*734A>T) identified in families affected by dominant glaucoma were cloned and functionally evaluated by miRNA target reporter and transactivation assays. Informed consent was obtained from the patients included in this study. The inform consent was approved by the Ethics Committee for Human Research of the Hospital Clínico San Carlos, Madrid (Spain), and followed the tenets of the Declaration of Helsinki.

\subsection{Cloning of FOXC1 and site directed mutagenesis}

The wild-type $\mathrm{FOXCl}$ coding sequence, obtained from a commercial cDNA (Origene, NM_001453), was subcloned into the EcoRI and BamHI restriction sites of the modified mammalian cell expression vector pcDNA3.1(-) (C-terminal Myc-Tagged) [3] by directional PCR using the following

primers: 5'GGGAATTCGGGGCCATGCAGGCGCGCTACT3' (P1) and 5'GTGGATCCCCAAACTTGCTACAGTCGTAGA3' (P2) (EcoRI and BamHI sequences are indicated in bold, respectively). This cDNA construct was used as a template for the site-directed mutagenesis of the p.I126S mutation using the QuickChange Site-directed Mutagenesis Kit (Stratagene), according to the manufacturer's instructions, and the following primer set: 5'GCTGGCAGAACAGCAGCCGCCACAACCTCTCG3' (forward) 5'CGAGAGGTTGTGGCGGCTGCTGTTCTGCCAGC3', (reverse). The p.G447_G448insDG variant was directly amplified from the genomic DNA of one carrier diagnosed with adult-onset dominant glaucoma (I:2, Fig. 1B) by directional PCR using the primers P1 and P2. The obtained amplicon was cloned into the EcoRI/BamHI restriction sites of the modified vector pcDNA3.1(-). A commercial cDNA, containing $1.8 \mathrm{~Kb}$ of the wild-type 3'-UTR FOXCl sequence (Origene, SC216457), was used as a template for the sitedirected mutagenesis of the c.*734A>T and c.*736A $>\mathrm{T}$ mutations using the QuickChange Site-directed Mutagenesis Kit, and the primers and PCR conditions indicated in Table 1. The mutagenesis products were sequenced to confirm the presence of the mutation and were subcloned into the EcoRI and NotI restriction sites of the pMirTarget vector (Origene) to avoid undesirable mutations in the vector. In order to obtain the mutant haplotypes p.I126S-c.*734A>T and p.H446_G447insGD-c.*734A>T, the cloned mutant 3'-UTR sequence was amplified using the following primers, which contained the HindIII restriction site indicated in bold: 5'GCAAGAAGCTTCACACCCTCAAAGCCG3' (forward) 5'CTCAAGCTTGGAGCGGCAGATAGCAGG3' and

(reverse). The PCR product was fused to each mutant coding region, previously cloned into the pcDNA3.1(-) plasmid, using the HindIII restriction site. The FOXCl wild-type coding sequence and 3'-UTR were also fused following a similar approach.

\section{2. miRNA target reporter assays}

The putative miRNA target sequences in the region surrounding the FOXCl c.*734A>T mutation, were identified using the MicroSNiPer software (http://cbdb.nimh.nih.gov/microsniper) [16]. To analyze the possible effect of this variant, the 1.8-Kb FOXCl 3'-UTR sequence (Origene, SC216457) was fused to the 3 '-end of the firefly luciferase coding region (cloned into the pMirTarget vector; OriGene, PS100062), using the SgfI/MluI restriction sites. This cDNA was used as a template to obtain the variants by site-directed mutagenesis, as described above. The miRNA target reporter assays were performed in triplicate in 24-well plates. The HEK-293T cells were transfected with $250 \mathrm{ng}$ of each 3'-UTR FOXCl target construct (wild-type or mutant) and $500 \mathrm{ng}$ of the recombinant $\mathrm{pCMV}$-MIR vector encoding either hsa-miR5481 (SC400522, Origene) or hsa-miR-548h (SC400512, Origene) using the Superfect Transfection Reagent according to the manufacturer's protocol. After a $48 \mathrm{~h}$ incubation, the luciferase activity was measured using the Luciferase Assay System (Promega) according to the manufacturer's instructions. RFP and LDH were detected via western blot as the transfection and loading controls, respectively, quantitated by densitometry $(n \geq 3)$, and significant differences were analyzed using the $t$-test.

\subsection{Transactivation assays}

The FOXC1 transactivation assays were performed using the luciferase assay previously indicated. A 600-bp fragment of the human CXCR4 gene promoter, which contains one FOX-binding element (FBE) ${ }^{17}$, was cloned into the NheI/NcoI restriction sites of the pGL3-basic vector (Promega) via directional PCR using normal human genomic DNA as a template and the following primers: 5'TCTGGCTAGCGCGCGGGGAATGGCGTTGG3' and 5'CTCCATGGTAACCGCTGGTTCTCCAG3' (the NheI and NcoI sequences are indicated with bold letters, respectively). HEK-293T cells in 24-well tissue culture plates $\left(2.5 \times 10^{5}\right.$ cells/well $)$ were transfected with $500 \mathrm{ng}$ of the FOXC1 expression vector, along with $50 \mathrm{ng}$ of the recombinant $\mathrm{pGL} 3$-basic-CXCR4 luciferase reporter, and 50 ng of the pMirTarget vector (Origene), which expresses red fluorescent protein (RFP) as a transfection control. The total 
amount of transfected DNA was equalized using the empty vector. The transactivation assays were performed $48 \mathrm{~h}$ after the transfection. The cells were harvested and assayed for firefly luciferase activity as previously indicated. FOXC1, RFP and LDH were detected by Western blot as the expression, transfection and loading controls, respectively. The RFP and LDH bands were quantitated via densitometry $(\mathrm{n} \geq 3)$ and significant differences were analyzed using the $t$ test.

\subsection{Statistical analyses}

The statistical comparisons between the groups were performed using either the $t$-test or the one-way analysis of variance (ANOVA). The data were statistically processed using the SigmaStat 2.0 software (SPSS Science).

\section{RESULTS}

\subsection{Identification of the $c . * 734 A>T$ FOXC1 variant in dominant glaucoma}

In a previous genetic study on dominant glaucoma we identified two probands who carried the FOXCl mutations p.I126S and p.G447_G448insDG along with the non-coding mutation c.*734A>T in the same gene (Escribano, personal communication). The three variants were present in the heterozygous state and the segregation analysis showed that the two coding mutations were coupled with the c.*734A $>\mathrm{T}$ mutation, forming two rare haplotypes (p.I126S—c.*734A $>\mathrm{T}$ and p.G447_G448insDG_c.*734A>T) (Fig. 1). The 3'-UTR variant was absent in 348 control subjects (696 chromosomes). The first haplotype is associated with remarkable phenotypic variability, ranging from isolated congenital and juvenile glaucoma to glaucoma with Axenfeld-Rieger anomaly, whilst the second haplotype is present in primary open-angle glaucoma cases diagnosed in their fifties or sixties (Escribano, personal communication).

\section{2. miRNA assay}

Previous bioinformatic analysis of the FOXCl 3'UTR sequence with the MicroSNiPer software showed that the variation c. $* 734 \mathrm{~A}>\mathrm{T}$ localized to a putative seed target sequence for hsa-miR-5481 (Fig. 2A) This nucleotide sequence was highly conserved in the four analysed mammals (Fig. 2B). To assess whether the c.*734A>T mutation is in fact embedded in the target sequence for this miRNA, the wild-type 3'-UTR and two 3'-UTR versions containing the c.*734A>T or c. $* 736 \mathrm{~A}>\mathrm{T}$ (control) mutations, were coupled to the 3 ' end of the luciferase coding region. A transient co-expression of the wild-type 3'UTR construct with cDNAs encoding either hsa-miR-5481 or hsa-miR-548h (negative miRNA control) was followed by an in vitro luciferase activity assay. hsa-miR-548h was used as a negative control because its target sequence was inferred to map near the mutation site (Fig. 2A). The results showed that the first miRNA reduced the wild-type 3'-UTRassociated luciferase activity to $80 \%$ of the control assay (Fig. 3, wild-type). In contrast, and as expected, the control
miRNA had no significant effect on luciferase activity (Fig. 3 , wild-type). The two mutant 3'-UTR sequences did not respond to the miRNAs used in the assays (Fig. 3, c.*734A>T and c.*736A>T). These results support the presence of a functional hsa-miR-5481 target sequence in the 3'-UTR of FOXCl and shows that its disruption by c. $* 734 \mathrm{~A}>\mathrm{T}$ specifically eliminates the effect of this miRNA on luciferase activity, showing that it is a loss-of-function mutation. Western blot analyses of RFP and LDH showed no significant differences in the transfection efficiency or sample loading, respectively (Fig. 3).

\subsection{Transcriptional activity}

To evaluate the effect of the c.*734A $>$ T variant on FOXC1 transactivation, the mutant 3'-UTR sequence was fused to the wild-type $\mathrm{FOXCl}$ coding sequence. In addition, cDNA constructs encoding the two FOXCl haplotypes identified in glaucoma patients were also obtained. Each of the individual cDNA constructs was transiently cotransfected with the luciferase reporter gene coupled to the CXCR4 promoter region, which contains one FOXC1 binding element. In addition, FOXC1 levels were estimated by western blot and quantitated by densitometry. According to the luciferase results described in the previous section, the variant c.*734A $>$ T, coupled to the wild-type FOXC1 coding sequence exhibited approximately 1.3-fold increased transactivation, compared to the wild-type construct (Fig. 4A). As expected, the cDNA constructs encoding either the previously known hypermorphic p.G447_G448insDG or hypomorphic p.I126S (Escribano, personal communication), variants, coupled to the wild-type 3'UTR sequence showed, respectively, a $150 \%$ increased or $20 \%$ decreased transactivation compared to the control (Fig. 4A). The two pathogenic haplotypes (p.G447_G448insDG-c.*734A>T and p.I126S - c.*734A>T) showed higher transactivation than the corresponding isolated coding mutation coupled to the wild-type 3'UTR sequence (approximately 1.2- to 1.3fold (Escribano, personal communication) on average; Fig. 4A). The FOXC1 protein levels associated with each of these cDNA constructs correlated with FOXC1 transactivation values (Fig. 4B), further supporting that $\mathrm{c} . * 734 \mathrm{~A}>\mathrm{T}$ disrupts the hsa-miR5481 target sequence leading to improved mRNA stability and thus, to increased protein and transactivation levels. Western blot analyses of RFP and LDH were also used as controls for the transfection efficiency and sample loading, respectively, and revealed no significant differences.

\section{DISCUSSION}

In previous studies we identified the c.*734A>T (rs35717904) mutation in two families affected by dominant glaucoma and in one patient diagnosed with non-dominant unilateral late-onset congenital glaucoma at the age of 5 years (Escribano, personal communication). In this study we have evaluated the role of this variant on both FOXC1 activity and glaucoma development. c.*734A $>\mathrm{T}$ has been described at low frequencies in Europeans (Ensembl variation database) and was not found in 696 control chromosomes, showing that it is an infrequent variant. 
Bioinformatics analyses of the c.*734A $>\mathrm{T}$ mutation led to the identification of hsa-miR5481 as a putative miRNA involved in regulating FOXCl translation (Escribano, personal communication). Our functional analyses confirmed that disruption of the hsa-miR5481 target sequence by the c. $* 734 \mathrm{~A}>\mathrm{T}$ mutation results in is a loss-of-function of this regulatory sequence, leading to improved mRNA stability and therefore, to moderately increased protein and transactivation levels. The sequence of the hsa-miR548-1 target was present in the four mammalian FOXCl genes analysed, indicating a relatively recent evolutionary origin and that this miRNA may regulate $F O X C l$ gene expression in these species. Currently, more than 120 members of the hsa-miR548 gene family are recognized (miRBase, release 21; http://www.mirbase.org/), which regulate to more than 3500 putative target genes [18]. This gene family is primate specific and is derived from Made1 transposable elements, and cancer-related regulatory roles have been suggested for hsa-mir-548 [18]. Our results also support that one member of this family, mir-5841, participates in FOXC1 translation regulation, contributing to understand the mechanisms involved in FOXCl expression regulation at the posttranscriptional level. It is known that FOXC1 expression is regulated by miR-204, which binds to two target sequences in the 3'-UTR and it has been proposed a crucial role for miR-204-FOXC1 interaction in endometrial cancer progression [8]. Moreover, our study supports that dysregulation of mir-5841 plays a role in glaucoma as a modifier factor for the activity of other two FOXC1 mutations, being one of only a few miRNAs known to be related with this disease. Dysregulation of miRNAs and their targets has the potential to be pathogenic, not only as disease causing genes but also as modifier factors [19]. In this line, the two pathogenic haplotypes identified in this study, which include the c. ${ }^{* 734 A}>\mathrm{T}$ variant (p.I126S — c. $* 734 \mathrm{~A}>\mathrm{T}$ and p.G447_G448insDG—c.*734A>T), showed higher transactivation than the individual coding mutations. Therefore, the loss-of-function associated with p.I126S was partially balanced by the presence of c. $* 734 \mathrm{~A}>\mathrm{T}$, leading to improved mRNA stability, increased protein levels and higher transactivation than the single p.I126S mutation. This increased activity might explain why haplotype p.I126S — c. $* 734 \mathrm{~A}>\mathrm{T}$ is associated with glaucoma, whilst the single related mutation p.I126M, whose reduced activity is similar to that of individual p.I126S (Escribano, personal communication), has been found associated with ARS and glaucoma [6]. p.I126M has also been proposed to be a positive control mutant for activity studies in the forkhead family of transcription factors [7]. Decreased transactivation of both p.I126S and p.I126M mutations has been attributed to impaired DNA binding affinity, probably due to structural alterations in the forkhead domain alpha-helix 3, which is predicted to play a role in major DNA groove recognition. Although protein phosphorylation and subcellular localization differences between these two mutations have been reported, these functional alterations do not correlate with the severity of the associated phenotypes. Therefore, the modifier effect of c. $* 734 \mathrm{~A}>\mathrm{T}$ on p.I126S and
p.G447_G448insDG FOXC1 mutations may contribute to the final phenotypic difference. Regarding pathogenicity of p.G447_G448insDG, it has been proposed that its increased transcription activity may depend on a gain-of-function that results in altered binding to transcription factors (Escribano, personal communication). Sequence analysis of the FOXCl 3'-UTR in glaucoma patients carrying coding FOXCl mutations may contribute to confirm the role of $\mathrm{c} . * 734 \mathrm{~A}>\mathrm{T}$ as a modifier factor in this disease.

FOXCl mutations are known to be associated with remarkable phenotypic variability [20,21], which suggests an important role for modifier factors (genetic, environmental and/or stochastic) on the phenotypic outcomes. According to the previously formulated hypothesis of critical activity thresholds for FOXC1 and the related protein PITX2 [22], we have proposed that the phenotypic variability associated with FOXCl mutations may depend on narrow intervals of residual transactivaton related to the individual FOXCl genotypes (Escribano, personal communication). In this conceptual framework, the modifier effect of the c. $* 734 \mathrm{~A}>\mathrm{T}$ variant may contribute to the overall genotypic-associated transactivation that influence the final phenotypic outcome.

\section{CONCLUSION}

This study shows that hsa-miR548-1 regulates FOXC1 translation, contributing to better understand the fine regulation of the biological function of this transcription factor. Our data also support that the c.*734A>T FOXC1 mutation disrupts the seed target sequence of this miRNA, resulting in a loss-of function and acting as a modifier factor for the activity of other FOXCl mutations. This modifier effect may help to explain the phenotypic variability observed in FOXCl-associated glaucoma. Finally, the identification of new miRNAs as regulators of FOXC1 translation provides new potential therapeutic targets to correct altered FOXC1 levels, which are present in diseases ranging from Axenfeld-Rieger malformations to isolated dominant glaucoma.

\section{CONFLICT OF INTEREST}

The authors confirm that this article content has no conflicts of interest.

\section{ACKNOWLEDGEMENTS}

This study has been supported by research grants from the Regional Ministry of Health (GCS-2006_C/12), the Regional Ministry of Science and Technology of the Board of the Communities of "Castilla-La Mancha" (PAI-05-002 and PCI08-0036), and the "Instituto de Salud Carlos III" (RD07/0062/0014, RD12/0034/0003 and PI11/00662). We are grateful to Mrs. María-José Cabañero for excellent technical assistance.

\section{REFERENCES}

[1] Saccà SC, Izzotti A: Oxidative stress and glaucoma: injury in the anterior segment of the eye. ProgBrainRes 2008; 173: 385-407. 
[2] Ferreira SM, Lerner SF, Brunzini R, Evelson PA, Llesuy SF: Oxidative stress markers in aqueous humor of glaucoma patients. AmJOphthalmol 2004; 137: 62-69.

[3] Lopez-Garrido MP, Medina-Trillo C, Morales-Fernandez $\mathrm{L}$, et al. Null CYP1B1 genotypes in primary congenital and nondominant juvenile glaucoma. Ophthalmology 2013; 120: 716-723.

[4] Campos-Mollo E, Lopez-Garrido MP, Blanco-Marchite $\mathrm{C}$, et al. CYP1B1 mutations in Spanish patients with primary congenital glaucoma: phenotypic and functional variability. MolVis 2009; 15: 417-431.

[5] Lines MA, Kozlowski K, Walter MA. Molecular genetics of Axenfeld-Rieger malformations. HumMolGenet 2002; 11: 1177-1184.

[6] Nishimura DY, Swiderski RE, Alward WL, et al. The forkhead transcription factor gene FKHL7 is responsible for glaucoma phenotypes which map to $6 \mathrm{p} 25$. NatGenet 1998; 19: 140-147.

[7] Saleem RA, Banerjee-Basu S, Berry FB, Baxevanis AD, Walter MA. Analyses of the effects that disease-causing missense mutations have on the structure and function of the winged-helix protein FOXC1. AmJHumGenet 2001; 68: 627-641.

[8] Chung TK, Lau TS, Cheung TH, et al. Dysregulation of microRNA-204 mediates migration and invasion of endometrial cancer by regulating $\mathrm{FOXC1}$. Int $\mathrm{J}$ Cancer 2012; 130: 1036-1045.

[9] Lin Z, Sun L, Chen W, et al. miR-639 regulates transforming growth factor beta-induced epithelialmesenchymal transition in human tongue cancer cells by targeting FOXC1. Cancer Sci 2014; 105: 1288-1298.

[10] Paylakhi SH, Moazzeni H, Yazdani S, et al. FOXC1 in human trabecular meshwork cells is involved in regulatory pathway that includes miR-204, MEIS2, and ITG $\beta 1$. Exp Eye Res 2013; 111: 112-121.

[11] Holley CL, Topkara VK.. An introduction to small noncoding RNAs: miRNA and snoRNA. Cardiovasc Drugs Ther 2011; 25: 151-159.

[12] Stefani G, Slack FJ. Small non-coding RNAs in animal development. Nat Rev Mol Cell Biol 2008; 9: 219-230.

[13] Luna C, Li G, Huang J, et al. Regulation of trabecular meshwork cell contraction and intraocular pressure by miR-200c. PLoS One 2012; 7: e51688.

[14] Li G, Luna C, Qiu J, Epstein DL, Gonzalez P. Role of miR-204 in the regulation of apoptosis, endoplasmic reticulum stress response, and inflammation in human trabecular meshwork cells. Invest Ophthalmol Vis Sci 2011; 52: 2999-3007.

[15] Blanco-Marchite C, Sanchez-Sanchez F, Lopez-Garrido $\mathrm{MP}$, et al. WDR36 and P53 gene variants and susceptibility to primary open-angle glaucoma: analysis of gene-gene interactions. Invest OphthalmolVisSci 2011; 52: 8467-8478.

[16] Barenboim M, Zoltick BJ, Guo Y, Weinberger DR. MicroSNiPer: a web tool for prediction of SNP effects on putative microRNA targets. HumMutat 2010; 31 : 1223-1232.

[17] Hayashi H, Kume $\mathrm{T}$ Forkhead transcription factors regulate expression of the chemokine receptor CXCR4 in endothelial cells and CXCL12-induced cell migration. BiochemBiophysResCommun 2008; 367: 584-589.

[18] Piriyapongsa J, Jordan IK. A family of human microRNA genes from miniature inverted-repeat transposable elements. PLoS One 2007; 2: e203.

[19] Bandiera S, Hatem E, Lyonnet S, Henrion-Caude A. microRNAs in diseases: from candidate to modifier genes. Clin Genet 2010; 77: 306-313.

[20] Komatireddy S, Chakrabarti S, Mandal AK, et al. Mutation spectrum of FOXC1 and clinical genetic heterogeneity of Axenfeld-Rieger anomaly in India. MolVis 2003; 9: 43-48.

[21] Perveen R, Lloyd IC, Clayton-Smith J, et al.Phenotypic variability and asymmetry of Rieger syndrome associated with PITX2 mutations. Invest OphthalmolVisSci 2000; 41: 2456-2460.

[22] Walter MA. PITs and FOXes in ocular genetics: the Cogan lecture. Invest OphthalmolVisSci 2003; 44: 1402-1405.

[23] Thompson JD, Higgins DG, Gibson TJ. CLUSTAL W: improving the sensitivity of progressive multiple sequence alignment through sequence weighting, position-specific gap penalties and weight matrix choice. Nucleic Acids Res 1994; 22: 4673-4680. 


\section{FIGURE LEGENDS}

Fig. (1). FOXCl haplotype segregation in two families affected by autosomal dominant glaucoma. Pedigrees of families PCG73 (A) and 556-10 (B). The variants in the two vertical lines represent the haplotypes. The black, dark grey and light grey symbols indicate congenital glaucoma, juvenile glaucoma and adult-onset primary open-angle glaucoma, respectively. +: wild-type allele. insDG: p.G447_G448insDG. The complete pedigrees have previously been reported (Escribano, personal communication).

Fig. (2). (A) The c.*734A>T FOXC1 mutation disrupts a putative target sequence for hsa-miR-5481. The target miRNAs were predicted with the MicroSNiPer software. The c. ${ }^{*} 734 \mathrm{~A}>\mathrm{T}$ mutation maps at the predicted target sequence for hsa-miR-5481. The arrow indicates the position of the mutant nucleotide. The predicted binding site of the control hsa-miR548h is also indicated. Note that the c.*734A $>\mathrm{T}$ mutation does not affect the interaction of this control miRNA with its target site. The asterisk indicates the position of the c.*736A>T mutation, a control of the seed sequence. Vertical lines and dots in the predicted miRNA target sequence indicate perfect Watson-Crick and G:U wobble pairings, respectively. The discontinuous vertical line indicates the base pairing between the miRNA and the wild-type target sequence, which is disrupted by the mutant nucleotide. (B) Comparison of the hsa-miR-5481 target nucleotide sequence in the FOXCl 3'-UTR from different species. The box shows the target sequence and the underlined nucleotides correspond to the seed sequence. The alignments were carried out with ClustalW [23]. The grey background indicates the positions where the nucleotides are identical.

Fig. (3). The c.*734A $>\mathrm{T}$ FOXC1 mutation specifically diminish hsa-miR-5481-dependent luciferase activity. Influence of hsa-miR-5481 and hsa-miR548h on luciferase activity associated to mutations. HEK-293T cells were cotransfected with each of the different 3'-UTR FOXC1 target constructs (wild-type, c.*734A>T or control c.*736A>T), fused to the $3^{\prime}$ end of the firefly luciferase coding region in the pMIRTarget vector, and the pCMV-MIR vector encoding either hsa-miR-5481 or hsa-miR-548h. After $48 \mathrm{~h}$ of incubation, luciferase activity was measured as described in the Materials and Methods. RFP and LDH were detected via western blot as transfection and loading control, respectively. Error bars correspond to the SD of three independent experiments carried out in triplicate. Asterisks indicate statistical significance: $\mathrm{p}<0.001(* * *)$. Significance was calculated by the one-way ANOVA test.

Fig. (4). The c. ${ }^{*} 734 \mathrm{~A}>\mathrm{T}$ FOXC1 variants modifies both transactivation and protein levels of two coding mutations. (A) cDNA constructs encoding the indicated FOXCl variants were transiently co-expressed with the reporter cDNA (pGL3-basic-CXCR4 promoter-luciferase) in HEK293T cells. The transactivation activity, expressed as a percentage of the luciferase activity of the wild-type protein, was measured as indicated in the Materials and Methods. The protein levels of the different FOXC1 versions present in HEK-293T cells $48 \mathrm{~h}$ after transfection were determined by western immunoblot using a monoclonal anti-myc antibody (Santa Cruz). Each lane contained $15 \mu \mathrm{g}$ of total protein obtained from the cell lysates. Transfection efficiency was assessed via western immunoblot using an anti-RFP antibody (Evrogen). The sample loading control, endogenous LDH, was also detected via immunoblot using an anti-LDH antibody (Chemicon). (B) FOXC1 levels quantitated by densitometry from bands obtained by western blot. Error bars correspond to the SD of three independent experiments carried out in triplicate. insDG: p.G447_G448insDG. NT: non-transfected cells (negative control). Asterisks indicate statistical significance: $\mathrm{p}<0.05$ $(*) ; \mathrm{p}<0.01(* *) ; \mathrm{p}<0.001(* * *)$. Significance was calculated by the one-way ANOVA test. 
Fig. (1).

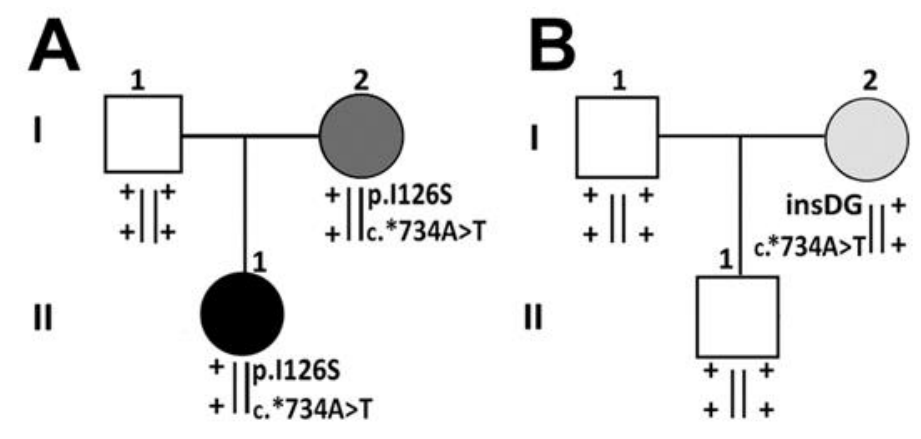

Fig. (2).

$\mathbf{A}$

$$
\begin{array}{r}
\text { hsa-miR-5481 } \\
\text { c. }{ }^{7} 734 \mathrm{~A}>\mathrm{T} \\
\text { hsa-miR-548h }
\end{array}
$$

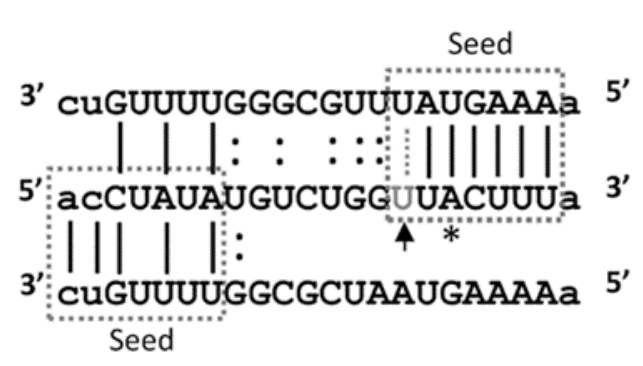

$$
\text { c. } * 734 \mathrm{~A}>\mathrm{T}
$$

$B H$. Sapiens CAGTTTGAATGAGAOCTATATGTCTGGATACTTTAATAGAGCT-TTAATTATTACGAAAA

M. Mulatta CAGTTTGAATGAGAdCTATATGTCTGGATACTTTAATAGAGCT-TTAATTATTACGAAAA M. Musculus CAGTTTGAATAAGAOCTATATGTCTGAATACTTMAATACAGCT-TTAAT-A----GAAAA R. Norvegicus CAGTTTGAATAAGAOCTATATGTCTGAATACTTTAATATAGCT-TTAAT-ATAÄGGAAAA

$X$. Laevis -AATTTAA---AGAA-------------------AAGCTGCCGAT-------GCAA T. Rubripes -AATCTGA-CAAAGCG-CGTTTCTTGCÄACCTGAATGTGGA--TTAATCGTCÄTCGTAG 
Fig. (3).

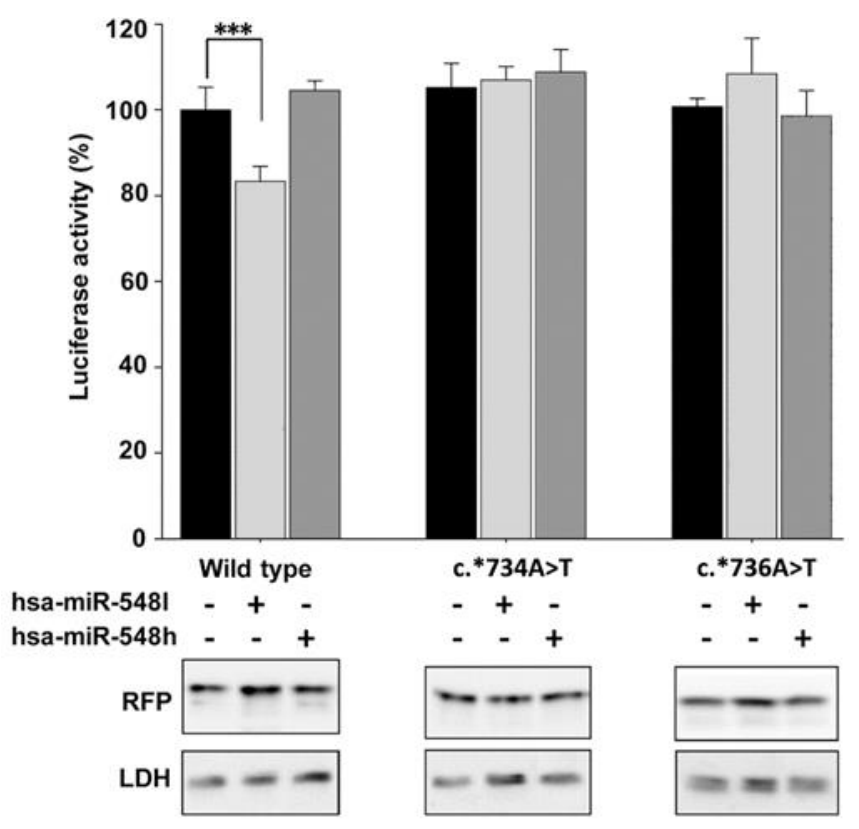

Fig. (4).

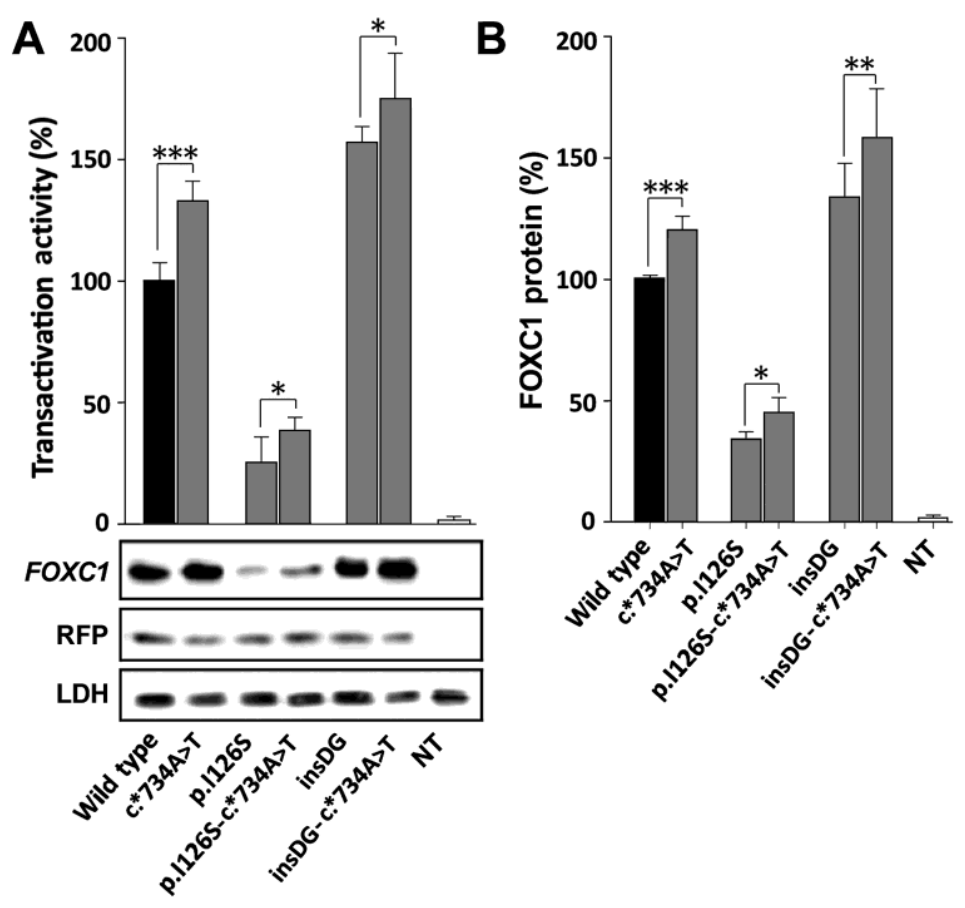


Table 1. The primer sequences and PCR conditions used for FOXC1 site-directed mutagenesis

\begin{tabular}{llcc}
\hline Primer set & \multicolumn{1}{c}{ Sequence $\left(\mathbf{5}^{\prime} \rightarrow \mathbf{3}^{\prime}\right)$} & $\begin{array}{c}\text { Annealing } \\
\text { temperature } \\
\left({ }^{\circ} \mathbf{C}\right) / \text { time }(\mathbf{s})\end{array}$ & $\begin{array}{c}\text { PCR } \\
\text { cycles }\end{array}$ \\
c. $* 734 A>T$ & F: GAATGAGACCTATATGTCTGGTTACTTTAATAGAGCTTTAATTAT & $58 / 15$ & 30 \\
& R: ATAATTAAAGCTCTATTAAAGTAACCAGACATATAGGTCTCATTC & & \\
c. $* 736 A>T$ & F: GAATGAGACCTATATGTCTGGATTCTTTAATAGAGCTTTAATTAT & $58 / 15$ & 30 \\
& R: ATAATTAAAGCTCTATTAAAGAATCCAGACATATAGGTCTCATTC & &
\end{tabular}

F: forward; R: reverse. The mutant nucleotides are indicated in bold. 